\title{
ARTICLE
}

\section{High prevalence of ischaemia, infection and serious comorbidity in patients with diabetic foot disease in Europe. Baseline results from the Eurodiale study}

\author{
L. Prompers • M. Huijberts • J. Apelqvist • E. Jude • \\ A. Piaggesi $\cdot$ K. Bakker $\cdot$ M. Edmonds $\cdot$ P. Holstein • \\ A. Jirkovska • D. Mauricio • G. Ragnarson Tennvall • \\ H. Reike • M. Spraul • L. Uccioli • V. Urbancic • \\ K. Van Acker • J. van Baal • F. van Merode $\cdot$ N. Schaper
}

Received: 22 July 2006 / Accepted: 14 August 2006 / Published online: 9 November 2006

(C) Springer-Verlag 2006

\begin{abstract}
Aims/hypothesis Large clinical studies describing the typical clinical presentation of diabetic foot ulcers are limited and most studies were performed in single centres with the possibility of selection of specific subgroups. The aim of this study was to investigate the characteristics of diabetic
\end{abstract}

L. Prompers $(\bowtie) \cdot$ M. Huijberts $\cdot$ N. Schaper

Department of Internal Medicine, University Hospital Maastricht,

P. Debeyelaan 25, P.O. Box 5800, 6202 AZ Maastricht,

The Netherlands

e-mail: Leonne.Prompers@intmed.unimaas.nl

J. Apelqvist

Department of Endocrinology, University of Malmö,

Malmö, Sweden

E. Jude

Diabetes Centre, Tameside General Hospital,

Ashton-under-Lyne, UK

\section{A. Piaggesi}

Diabetic Foot Unit, Department of Endocrinology

and Metabolism, Azienda Pisa University Hospital,

Pisa, Italy

K. Bakker

IDF Consultative Section, International Working Group

on the Diabetic Foot,

Heemstede, the Netherlands

\section{Edmonds}

Diabetic Department, King's College Hospital,

London, UK

P. Holstein

Copenhagen Wound Healing Centre, Bispebjerg Hospital,

Copenhagen, Denmark patients with a foot ulcer in 14 European hospitals in ten countries.

Methods The study population included 1,229 consecutive patients presenting with a new foot ulcer between 1 September 2003 and 1 October 2004. Standardised data on patient characteristics, as well as foot and ulcer

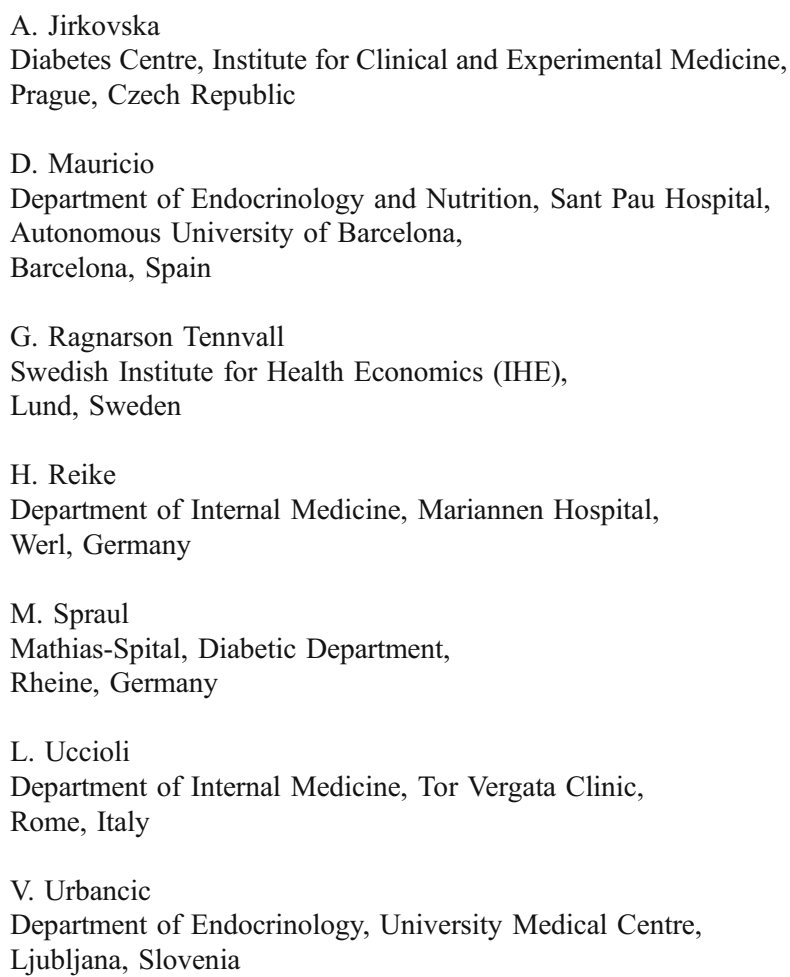

L. Uccioli

Department of Internal Medicine, Tor Vergata Clinic,

Rome, Italy

V. Urbancic

Department of Endocrinology, University Medical Centre, Ljubljana, Slovenia 
characteristics, were obtained. Foot disease was categorised into four stages according to the presence or absence of peripheral arterial disease (PAD) and infection: A: PAD -, infection -; B: PAD -, infection +; C: PAD +, infection -; $\mathrm{D}: \mathrm{PAD}+$, infection + .

Results PAD was diagnosed in $49 \%$ of the subjects, infection in $58 \%$. The majority of ulcers $(52 \%)$ were located on the non-plantar surface of the foot. With regard to severity, $24 \%$ had stage A, $27 \%$ had stage B, $18 \%$ had stage $\mathrm{C}$ and $31 \%$ had stage $\mathrm{D}$ foot disease. Patients in the latter group had a distinct profile: they were older, had more non-plantar ulcers, greater tissue loss and more serious comorbidity.

Conclusions/interpretation According to our results in this European cohort, the severity of diabetic foot ulcers at presentation is greater than previously reported, as one-third had both PAD and infection. Non-plantar foot ulcers were more common than plantar ulcers, especially in patients with severe disease, and serious comorbidity increased significantly with increasing severity of foot disease. Further research is needed to obtain insight into the clinical outcome of these patients.

Keywords Diabetes - Diabetic foot ulcer - Multicentre · Neuropathy $\cdot$ Peripheral arterial disease

\begin{tabular}{ll}
\multicolumn{2}{l}{ Abbreviations } \\
ABPI & ankle-brachial pressure index \\
Eurodiale & European Study Group on Diabetes and the \\
& Lower Extremity \\
NYHA & New York Heart Association \\
PAD & peripheral arterial disease
\end{tabular}

\section{Introduction}

One in every seven individuals with diabetes will suffer from a foot ulcer during their lifetime [1,2]; many of these ulcers will need intensive treatment and hospitalisation, healing often takes months, and some patients will eventually lose their leg. Given the high costs associated with these ulcers, this disease is not only a major burden to

\footnotetext{
K. Van Acker

Department of Endocrinology, St Joseph Clinic,

Bornen, Belgium

J. van Baal

Department of Surgery, Twenteborg Hospital,

Almelo, the Netherlands

F. van Merode

Department of Health Organisation, Policy and Economics,

University of Maastricht,

Maastricht, the Netherlands
}

the patient but also to the healthcare system [3], and there is growing awareness that more attention should be given to this condition [4]. The development of a foot ulcer usually involves several mechanisms, such as neuropathy, increased biomechanical stress, external trauma and peripheral arterial disease (PAD) [5]. Moreover, these ulcers are often complicated by infection [6]. Thus, different presentations of foot ulcers can be encountered in clinical practice, and management strategies, outcome and resource utilisation are strongly influenced by characteristics of these, such as the presence of PAD, the presence of infection and, probably, the presence of comorbidity $[1,7]$. The number of large studies reporting on patient and ulcer characteristics is limited, but significant differences in the underlying pathologies and patient characteristics have been reported $[1,8-10]$. These differences might be related to factors such as race and variation in demographics, but it is also likely that the characteristics of the local healthcare organisation will determine the type of patients that are reported on, owing to differences in referral. In many centres, patients with neuropathic or mildly neuroischaemic ulcers are treated in foot clinics, while patients with severe ischaemia and deep foot infections are admitted to surgical wards. From several studies there emerges a 'classic' picture of a patient with a neuropathic ulcer on the plantar surface of the foot $[1,8,11,12]$; with optimal therapy $>80 \%$ of these 'uncomplicated' ulcers can heal within 3 months [13-15]. One might therefore question whether some studies provide an image of diabetic foot disease that is biased towards the least complicated foot ulcers or a specific subset of patients.

The European Study Group on Diabetes and the Lower Extremity (Eurodiale) is a collaborative network of 14 European centres, originally created to stimulate multidisciplinary research in the field of diabetic foot disease. One of the main aims of the Eurodiale group is to obtain data on clinical outcome in patients with different ulcer phenotypes and disease severity to provide international reference data for healing rate and healing time, as well as data on adverse outcomes such as non-healing, amputation and death. In addition, the identification of the main determinants of outcome was an important objective of this study.

Therefore this multi-disciplinary group, comprising diabetologists, vascular surgeons and orthopaedic surgeons, initiated a prospective data collection study, in which baseline and 12-month follow-up data on all patients treated in these centres for a new foot ulcer were collected. The study was designed in such a way that data collection was possible during daily practice, in order to recruit a cohort as unselected as possible. This report provides an overview of the baseline characteristics of the Eurodiale cohort to present a picture of the severity of this disease in the 'average' European patient with a diabetic foot ulcer. 


\section{Subjects and methods}

\section{Study design}

All consecutive diabetic patients presenting with a new foot ulcer between 1 September 2003 and 1 October 2004 were asked to participate in the study; 1,229 patients attending 1 of 14 diabetic foot centres in ten European countries were included and followed until healing or major amputation or for the maximum period of 1 year. Follow-up visits were performed every 4 weeks. At baseline and during all follow-up visits, data were recorded on standardised case record forms. The baseline and follow-up forms were designed by the Eurodiale group during a 1-year preparatory phase and were tested in a pilot study. In total, 80 items were to be collected at baseline to describe the most important individual and disease-specific factors that might influence management strategies and outcome. All personnel involved in the data collection were trained on several occasions (including site visits) in the use of the case record form and the techniques required to obtain the data. All case record forms were sent to the primary study centre where they were checked by data managers. If there were missing data or inconsistencies, the participating centre was contacted to obtain additional information. Subsequently, the forms were scanned and transformed into ASCII files.

A foot ulcer was defined as a full-thickness lesion below the ankle. Exclusion criteria were treatment for an ulcer on the same foot during the 12 months before inclusion and a life expectancy shorter than 1 year. Both patients presenting with a foot ulcer at the outpatient clinic and those admitted to hospital were recruited, irrespective of the specialist primarily responsible for the patient's care. The mean number of patients per centre was 88 (range 40-126). Thirty-seven per cent of the included patients were referred by a medical specialist; the others were referred by their general practitioner or were self-referrals. The local ethics committees of the 14 hospitals approved the study protocol and all patients gave written informed consent.

\section{Patient and foot characteristics}

The patient characteristics recorded included comorbidity, living conditions and previous management. Disabling comorbidity was defined as the presence of severe visual impairment (defined as the inability to read a newspaper after correction), end-stage renal disease (defined as renal replacement therapy), heart failure and/or angina pectoris New York Heart Association (NYHA) classification III or IV, and/or the inability to stand or walk without help. Various techniques and definitions can be used to describe the underlying pathology of a foot ulcer to assess and classify its severity. In this study all patients underwent a standardised examination according to the PEDIS system, which classifies foot ulcers according to five categories: perfusion, extent, depth, infection and sensation $[16,17]$. This system was developed by the International Consensus on the Diabetic Foot for the classification of patients for clinical research purposes. Perfusion assessment followed PEDIS guidelines and included evaluation of the presence of pedal pulses and measurement of the ankle-brachial pressure index (ABPI) using a handheld Doppler device. The presence of PAD was defined as an ABPI of $<0.9$ and/ or two absent foot pulses [16]. In addition, we graded an ABPI of $<0.5$ as severe limb ischaemia. Extent was determined by multiplying the largest diameter by the second largest diameter perpendicular to the first [16]. Depth was described as superficial or deep: a superficial ulcer was a full-thickness lesion of the skin not extending through the subcutis, and a deep ulcer was a lesion of the skin extending through the subcutis. Infection was diagnosed if two or more of the following signs were present: frank purulence, local warmth, erythema, lymphangitis, oedema, pain, fever and foul smell. Evaluation of sensation (neuropathy) included pressure sensation (10 g monofilament on plantar aspect of hallux, metatarsophalangeal joint 1 and 5), tactile sensation (cotton wisp on the dorsum of the foot), vibration sensation (128 Hz tuning fork on the dorsum of the hallux) and blunt/sharp discrimination (dorsum of the foot) [16]. Neuropathy was diagnosed if the results of two or more of the aforementioned tests were abnormal [16]. In addition, the location of the ulcer was described (six predefined areas). In all patients, serum $\mathrm{HbA}_{1 \mathrm{c}}$ and serum creatinine levels were determined at each participating hospital.

\section{Classification of foot disease}

The presence of PAD and infection in patients with diabetic foot ulcers are major factors contributing to clinical outcome in terms of healing rates, healing time and amputation [8]. Foot ulcers were therefore categorised into four stages according to presence or absence of infection and PAD (Table 1); these stages are similar to those of the University of Texas system [8]. Using this classification, healing rates of $90 \%$ in stage A, $89 \%$ in stage B, $69 \%$ in stage $\mathrm{C}$ and $36 \%$ in stage $\mathrm{D}$ were reported [13].

\section{Statistical analysis}

Statistical analysis was performed using the SPSS statistical package, version 11.0 (SPSS, Chicago, IL, USA). As most data were scored as categories, the data are expressed as frequencies. Comparisons between groups were made using the $\chi^{2}$ test; statistical significance was defined as a $p$ value of $<0.05$. 
Table 1 Classification of foot disease

\begin{tabular}{llll}
\hline Stage & Definition & $\begin{array}{l}\text { Number of } \\
\text { patients }\end{array}$ & $\begin{array}{l}\text { Percentage of } \\
\text { study population }\end{array}$ \\
\hline A & PAD -, infection - & 270 & 24 \\
B & PAD -, infection + & 305 & 27 \\
C & PAD +, infection - & 205 & 18 \\
D & PAD +, infection + & 347 & 31 \\
\hline
\end{tabular}

\section{Results}

Patient characteristics

At the time of study entry, $27 \%$ of all patients were admitted to the hospital. Sixty-three per cent of the patients were either previously managed in primary care or did not receive any treatment before inclusion. General patient characteristics (Table 2) were similar in all centres. Disabling comorbidity was present in $32 \%$ of the patients: severe visual impairment in $15 \%$, end-stage renal disease in $6 \%$, heart failure and/or angina pectoris NYHA III/IV in $11 \%$, and the inability to stand or walk without help in $10 \%$ of all patients. Sixty per cent of the patients were not selfcaring but had a partner or relative who was involved in daily personal care; $18 \%$ of the patients were unemployed, $19 \%$ were employed and $64 \%$ were retired at study entry.

Ulcer and foot characteristics

Underlying pathology Among this cohort, PAD was present in $49 \%$; however this value is possibly an underestimation as $32 \%$ of the ABPIs were $>1.2$ and were therefore unreliable. PAD occurred more frequently in older patients (in $71 \%$ of patients aged $>70$ years) and in patients with disabling comorbidity $(60 \%)$. Severe limb ischaemia, defined as an ABPI of $<0.5$, was present in 113 patients (12\% of all patients). Diabetic neuropathy was diagnosed in $86 \%$; other neurological disorders resulting in loss of sensory or motor function (e.g. cerebrovascular accident) were present in $7 \%$.

Infection At the time of examination, $58 \%$ of the ulcers were infected; however, of the patients admitted to hos-

Table 2 General characteristics of the patients

\begin{tabular}{ll}
\hline Characteristic & \\
\hline Mean age (years) & $65 \pm 12$ \\
Men (\%) & 64 \\
$\mathrm{HbA}_{1 \mathrm{c}}>8.4 \%(\%)$ & 49 \\
Duration of diabetes $>10$ years (\%) & 70 \\
Disabling comorbidity (\%) & 32 \\
\hline
\end{tabular}

pital, $82 \%$ had evidence of infection. In patients with $\mathrm{PAD}$, infection was diagnosed more frequently (63 vs $53 \%$, $p<0.05)$.

Location, size, depth and duration Data on ulcer location are shown in Fig. 1. More than half of the ulcers (52\%) were non-plantar ulcers. The most frequent ulcer site was the dorsal or interdigital area of the toes (32\%). The classic plantar forefoot or midfoot ulcer was present in $22 \%$ of the patients. Almost half of the patients (45\%) had deep ulcers that extended into tissue below the subcutis (e.g. tendons, muscle or bone). Ulcer size was between 1 and $5 \mathrm{~cm}^{2}$ in the majority of the patients $(52 \%)$, and was $>5 \mathrm{~cm}^{2}$ in $11 \%$. The duration of the ulcer at study entry was shorter than 1 week in $16 \%$ of the patients, between 1 week and 3 months in $57 \%$, and $>3$ months in $27 \%$.

Patient and ulcer characteristics in relation to severity of disease Data on disease classification are shown in Table 1 and Fig. 2. Both infection and PAD were present in $31 \%$ of the patients (stage D). Ulcers in these patients were more often deep (64 vs $20 \%$ in stage A; $p<0.001$ ) and $>5 \mathrm{~cm}^{2}$ (20 vs $4 \%$ in stage A, $p<0.001$ ). Stage D ulcers were also more often non-plantar (65 vs $36 \%$ in stage A, $p<0.001$; Fig. 2a). Increasing disease severity was associated with age $(56 \%$ in stage $\mathrm{D}$ vs $22 \%$ in stage $\mathrm{A}$ were aged $>70$ years, $p<0.001$; Fig. 2b) and the prevalence of disabling comorbidity ( $38 \%$ in stage D vs $23 \%$ in stage A, $p<0.001$; Fig. 2c). The same pattern was observed for each comorbidity; Fig. $2 \mathrm{~d}$ shows the relationship between disease stage and visual impairment. The distribution of patients from stage A to D was similar for the group of referrals from primary care and the group referred by other specialists.

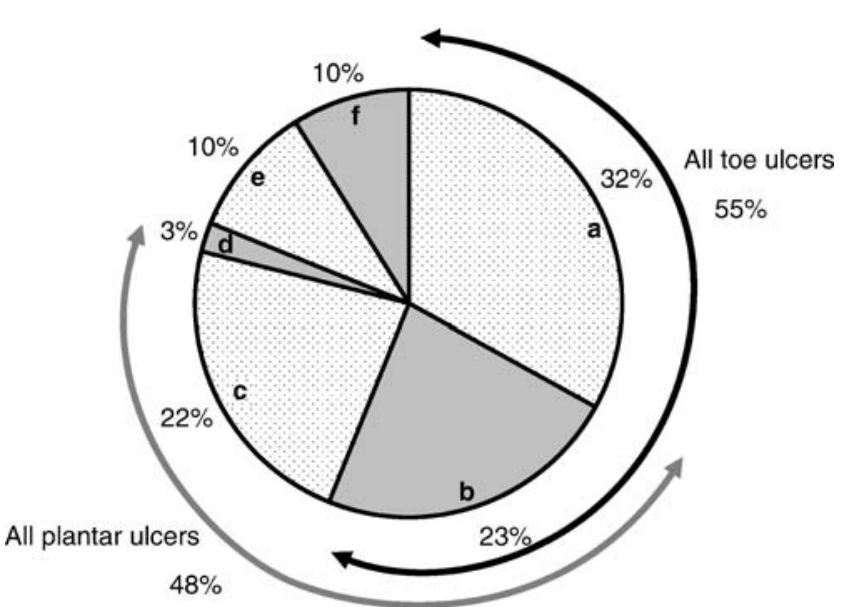

Fig. 1 a Dorsal/interdigital toes, b plantar toes, c plantar forefoot/ midfoot, d plantar hindfoot, e heel, f dorsal/lateral aspect foot 


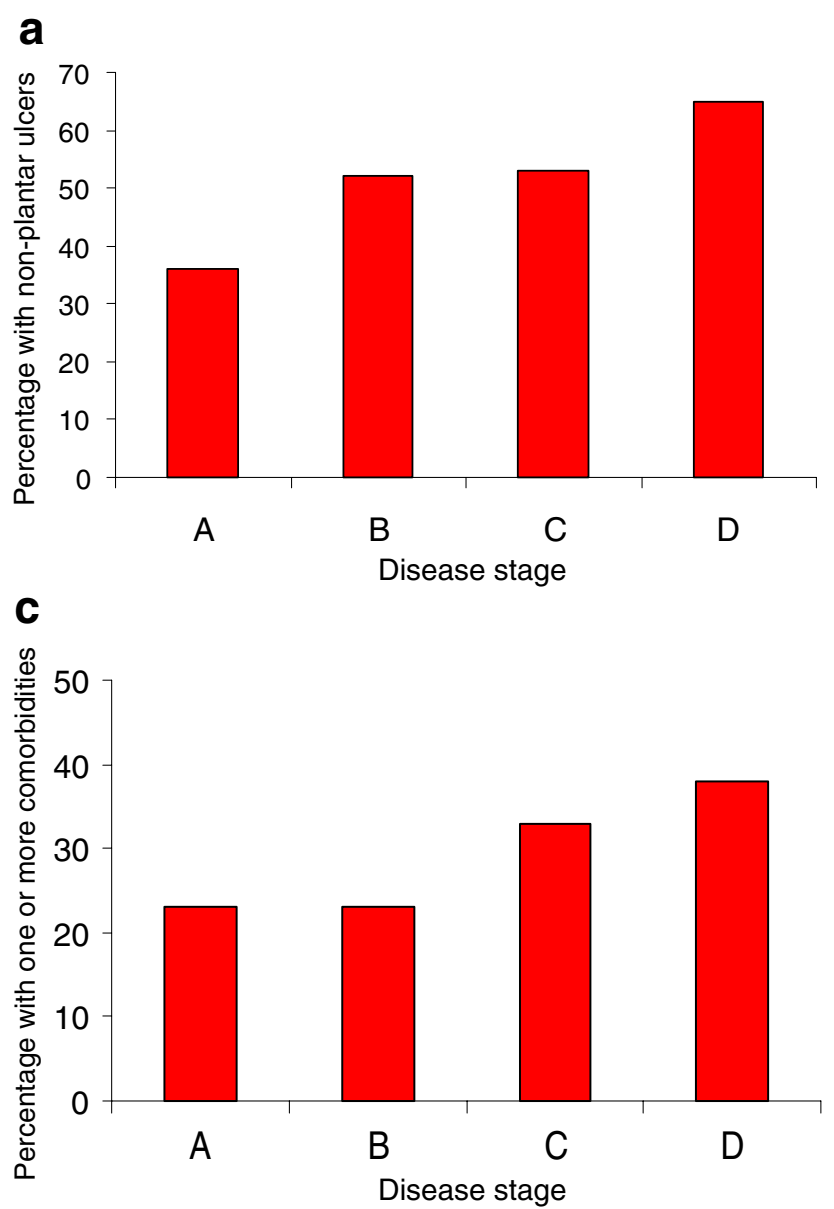

Fig. 2 Disease stage $(A: \mathrm{PAD}-$, infection $-; B$ : PAD -, infection + +; $\quad$ : PAD +, infection -; $D$ : PAD +, infection + ) vs the following ulcer and patient characteristics: a percentage with non-plantar ulcers,

\section{Discussion}

In this paper data are presented on disease severity and the relationship with patient characteristics in a cross-section of patients treated in clinics throughout Europe interested in the diabetic foot. Data were obtained in 1,229 diabetic patients with a new ulcer who were treated in 14 participating hospitals. One of the strengths of this study is that it reflects daily clinical practice. The main findings are that a large number of patients have severe disease (both infection and PAD were present in one-third of the patients); non-plantar foot ulcers are more common than plantar ulcers, especially in patients with severe disease; and serious comorbidity increases significantly with increasing severity of foot disease.

PAD and the presence of infection are well-known prognostic factors in diabetic foot disease [1], but how often do we encounter these problems in patients with foot ulcers? Knowledge of the frequencies of the different ulcer phenotypes is essential to predict outcome, to determine management strategies and to allocate resources. In patients

\section{b}
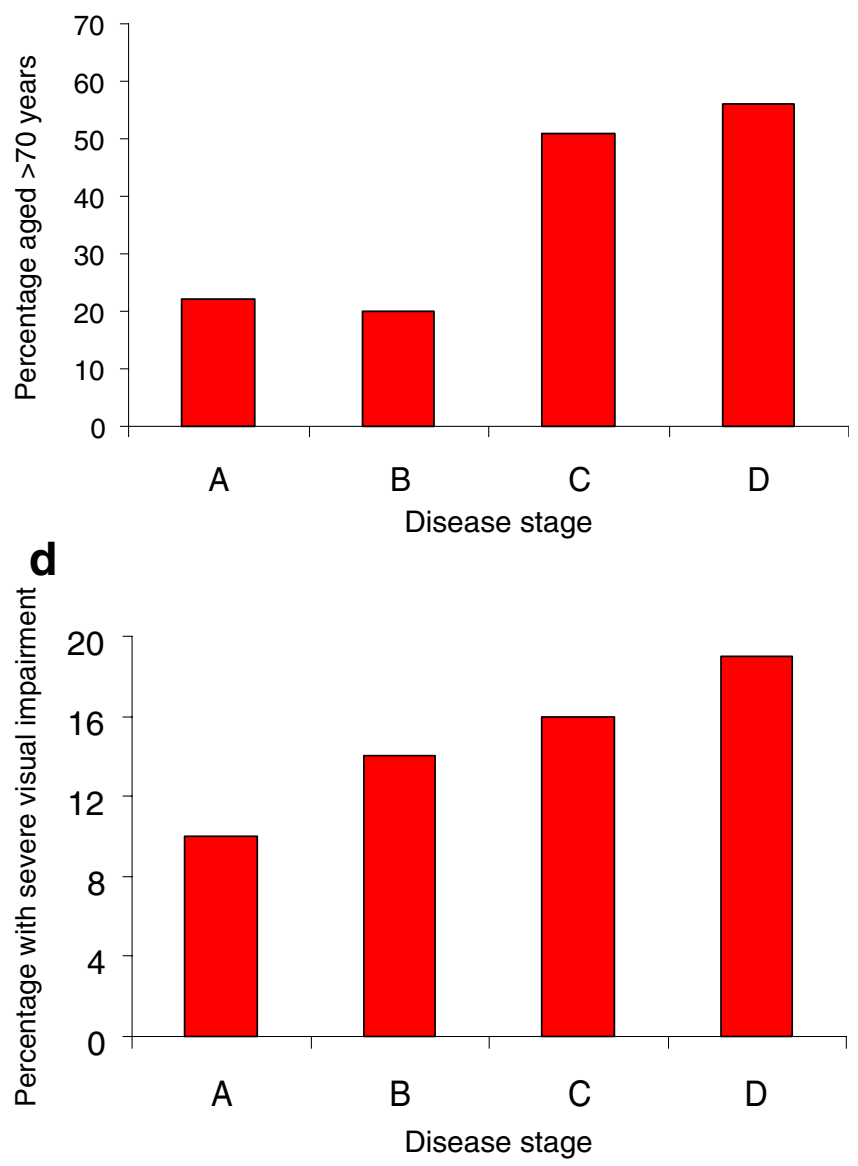

b percentage aged $>70$ years, c percentage with one or more comorbidities, d percentage with severe visual impairment

with diabetic foot ulcers the prevalence of PAD varies substantially between studies; indeed, in three studies that included up to 360 patients the reported prevalence ranged from 10 to $60 \%$ [8, 18-21]. In our study, PAD was present in $49 \%$ of patients, but varied from 22 to $73 \%$ in the different centres. This variation is difficult to explain and could be related to differences in the prevalence of PAD, but is more likely due to differences in healthcare organisation resulting in different referral patterns. These differences could also (in part) explain the differences in PAD prevalence in the aforementioned studies. Although our data indicate that PAD is common in foot ulcer patients, the degree of peripheral ischaemia can vary substantially between patients and, unfortunately, physical examination is of limited value [22]. In non-diabetic patients, measurement of the ABPI is the cornerstone of the evaluation of PAD [23]. However, in diabetic patients, non-compressibility of the lower leg arteries can occur as a result of media calcification (Mönckeberg's sclerosis), which produces a falsely elevated ABPI. Indeed, in our study, non-compressible lower leg arteries (defined as an ABPI of $>1.2$ ) were 
observed in $32 \%$ of the patients. As ABPI values below 0.9 are clearly indicative of PAD both in non-diabetic and diabetic patients, we are confident that these patients were correctly categorised as having PAD. However, the use of a more sensitive instrument would probably have resulted in an even higher prevalence of PAD in our patients with an $\mathrm{ABPI}$ of $>0.9$. Alternative techniques for diagnosis of $\mathrm{PAD}$, such as systolic toe pressure measurements or duplex scanning of the lower leg, were not available at all participating centres. Transcutaneous oxygen pressure can be used to predict the chance of healing of ischaemic foot ulcers [23, 24]; however, measurements are affected by the presence of neuropathy, infection and oedema [25]. Based on these considerations the combination of palpation of pulses and Doppler pressure measurement was chosen, as formulated by the International Consensus on the Diabetic Foot in their report on classification of foot ulcers for research purposes [16]. We also found also a relatively high prevalence of infection. In this study, $58 \%$ of all ulcers were infected at presentation according to predefined criteria; infection rates of $35-50 \%$ were reported in the majority of earlier studies [8, 18, 19].

In previous reports the combination of PAD and infection was associated with the poorest outcome. In the validation study of the University of Texas wound classification system [8], patients with the combination of infection and ischaemia were 90 times more likely to undergo a midfoot or higher amputation than those with less advanced wound stages. In the total contact cast study of Nabuurs-Franssen et al. [13], the healing rate of patients with mild to moderate ischaemia and infection was only $36 \%$, compared with a rate of $90 \%$ in patients with neuropathy with or without infection. Not many studies on patients with a foot ulcer report on the relative proportion of the study population with a high-risk profile. In three earlier studies these unfavourable disease characteristics were present in $5-15 \%$ of patients, whereas the proportion of our cohort with this profile was much higher $(31 \%)[8,18,19]$.

Our results clearly indicate that the ulcer and patient characteristics of this large group of patients with PAD and infection are different from those of patients with neither PAD nor infection. The ulcers in the former patients are mainly non-plantar (65\%) and are associated with more extensive tissue loss as they were also deeper and larger. It is evident that these patients are older and more frail, since serious comorbidity also clusters in this group.

Our findings have a number of implications. With regard to evidence-based management of diabetic foot ulcers, we might conclude that there is little evidence on how to treat the patients with the most severe disease, who make up a significant proportion of the patients seen in the foot clinic. Although relatively high rates of non-plantar foot ulceration
(40-50\%) have previously been observed $[1,26]$, earlier studies and guidelines focused on plantar foot ulceration in neuropathic patients. Examples include plantar pressure measurements [12], insoles for the prevention of plantar forefoot and midfoot ulcers [27], and total contact casting [14]. There are very few studies on the management of nonplantar ulcers, despite the fact that healing rates might be lower in dorsal compared with plantar ulcers [28]. Studies on the treatment of PAD in diabetic foot disease are also scarce, and many questions remain to be answered. It is unclear at what degree of peripheral ischaemia intervention is cost-effective and which procedure - bypass or endovascular intervention - should be performed [29, 30]. Treatment of infection in patients with PAD is another topic that has gained little attention. It has been shown that penetration of antibiotics is poorer in patients with PAD [31], but in several important trials on the treatment of infection in diabetic foot disease PAD was present in only a minority of patients $[32,33]$. The high number of patients with severe foot disease will also have implications for healthcare costs in view of the expected poor healing, the required diagnostic and interventional procedures, the use of antibiotics and the need for hospital admissions. Moreover, with regard to treatment, given the presence of significant comorbidities, a comprehensive, multidisciplinary approach is required. One may propose that allocation of resources should be adjusted to the relative proportion of patients with less severe (no PAD and no infection) vs severe disease (PAD plus infection). This study also shows that the patients with the poorest general health status have the most severe foot disease, and will need the most intensive therapy. This implies that the burden of disease is highest in those who are least able to cope with it. This should prompt caregivers to adapt management strategies to the specific characteristics of this group.

It is evident that this study has several limitations. It is a hospital-based observational study evaluating consecutive patients who were treated in European centres interested in diabetic foot care. The Eurodiale cohort included patients referred from a primary care setting or by other specialists, as well as patients not specifically referred. One-third of the patients were referred by other specialists, either from the same hospital or from other hospitals (tertiary referrals). These latter patients might therefore have had more severe disease, although in terms of ulcer stages, no differences were observed between patients who were referred by other specialists and those who were not. In addition, studies with long-term follow-up indicate that diabetic foot ulceration is a recurrent disease [34, 35]. Since we excluded patients with a previous ulcer within the 12 months prior to presentation, our cohort does not properly reflect the total population attending the participating clinics. Furthermore, since the study was embedded in daily clinical practice, 
limitations had to be set as to the number and type of data to be collected. The dataset does not give a complete description of all characteristics of these patients and, although based on current international guidelines, more sophisticated tools could have been used to assess certain pathologies.

In conclusion, these baseline data from the Eurodiale cohort, reflecting the average European patient with a diabetic foot ulcer, contain an important message: many patients with diabetic foot ulcers are severely ill, and this is reflected by the severe underlying pathology and the presence of disabling comorbidity. Follow-up data on these patients, which are expected in the coming year, could give us more insight into the implications of the severity of this disease for clinical outcome, resource utilization and quality of life.

Acknowledgements This study was supported by the fifth framework programme of the European Commission. We would like to thank all Eurodiale co-workers: M. Annersten, R. Bem, A. Boykowskov, H. Brill, S. Bus, A. De Leiva, J. De Neve, S. Di Cario, V. Fejfarova, J. Gaitan, D. Geenen, T. Geens, L. Giurato, B. Hempe, M. Hutten, J. Kersken, F. Palumbo, L. Rizzo, R. Roel, D. Simon, M. Slak.

Duality of interest We state that there is no duality of interest.

\section{References}

1. Reiber GE, Vileikyte L, Boyko EJ et al (1999) Causal pathways for incident lower-extremity ulcers in patients with diabetes from two settings. Diabetes Care 22:157-162

2. Reiber GE, Lipsky BA, Gibbons GW (1998) The burden of diabetic foot ulcers. Am J Surg 176:5S-10S

3. Ragnarson Tennvall G, Apelqvist J (2004) Health-economic consequences of diabetic foot lesions. Clin Infect Dis 39(Suppl 2): S132-S139

4. Jeffcoate WJ, Harding KG (2003) Diabetic foot ulcers. Lancet $361: 1545-1551$

5. Boulton AJ (2004) The diabetic foot: from art to science. The 18th Camillo Golgi lecture. Diabetologia 47:1343-1353

6. Frykberg RG (2003) An evidence-based approach to diabetic foot infections. Am J Surg 186:44S-54S; discussion 61S-64S

7. Apelqvist J, Agardh CD (1992) The association between clinical risk factors and outcome of diabetic foot ulcers. Diabetes Res Clin Pract 18:43-53

8. Armstrong DG, Lavery LA, Harkless LB (1998) Validation of a diabetic wound classification system. The contribution of depth, infection, and ischemia to risk of amputation. Diabetes Care 21:855-859

9. Ramsey SD, Newton K, Blough D et al (1999) Incidence, outcomes, and cost of foot ulcers in patients with diabetes. Diabetes Care 22:382-387

10. Margolis DJ, Allen-Taylor L, Hoffstad O, Berlin JA (2002) Diabetic neuropathic foot ulcers: the association of wound size, wound duration, and wound grade on healing. Diabetes Care 25:1835-1839

11. Mueller MJ, Sinacore DR, Hastings MK, Strube MJ, Johnson JE (2003) Effect of Achilles tendon lengthening on neuropathic plantar ulcers. A randomized clinical trial. J Bone Joint Surg Am 85-A:1436-1445

12. Lavery LA, Armstrong DG, Wunderlich RP, Tredwell J, Boulton AJ (2003) Predictive value of foot pressure assessment as part of a population-based diabetes disease management program. Diabetes Care 26:1069-1073

13. Nabuurs-Franssen MH, Sleegers R, Huijberts MS et al (2005) Total contact casting of the diabetic foot in daily practice: a prospective follow-up study. Diabetes Care 28:243-247

14. Mueller MJ, Diamond JE, Sinacore DR et al (1989) Total contact casting in treatment of diabetic plantar ulcers. Controlled clinical trial. Diabetes Care 12:384-388

15. Armstrong DG, Nguyen HC, Lavery LA, van Schie CH, Boulton AJ, Harkless LB (2001) Off-loading the diabetic foot wound: a randomized clinical trial. Diabetes Care 24:1019-1022

16. Schaper NC (2004) Diabetic foot ulcer classification system for research purposes: a progress report on criteria for including patients in research studies. Diabetes Metab Res Rev 20(Suppl 1): S90-S95

17. Boulton AJ, Gries FA, Jervell JA (1998) Guidelines for the diagnosis and outpatient management of diabetic peripheral neuropathy. Diabet Med 15:508-514

18. Oyibo SO, Jude EB, Tarawneh I et al (2001) The effects of ulcer size and site, patient's age, sex and type and duration of diabetes on the outcome of diabetic foot ulcers. Diabet Med 18: 133-138

19. Oyibo SO, Jude EB, Tarawneh I, Nguyen HC, Harkless LB, Boulton AJ (2001) A comparison of two diabetic foot ulcer classification systems: the Wagner and the University of Texas wound classification systems. Diabetes Care 24:84-88

20. Moulik PK, Mtonga R, Gill GV (2003) Amputation and mortality in new-onset diabetic foot ulcers stratified by etiology. Diabetes Care 26:491-494

21. Apelqvist J, Larsson J, Agardh CD (1990) The importance of peripheral pulses, peripheral oedema and local pain for the outcome of diabetic foot ulcers. Diabet Med 7:590-594

22. Schaper NC, Nabuurs-Franssen MH (2002) The diabetic foot: pathogenesis and clinical evaluation. Semin Vasc Med 2:221-228

23. Dormandy JA, Rutherford RB (2000) Management of peripheral arterial disease (PAD). TASC Working Group. TransAtlantic InterSociety Concensus (TASC). J Vasc Surg 31:S1-S296

24. Cavanagh PR, Lipsky BA, Bradbury AW, Botek G (2005) Treatment for diabetic foot ulcers. Lancet 366:1725-1735

25. Boyko EJ, Ahroni JH, Stensel VL, Smith DG, Davignon DR, Pecoraro RE (1996) Predictors of transcutaneous oxygen tension in the lower limbs of diabetic subjects. Diabet Med 13:549-554

26. Larsen K, Holstein P, Deckert T (1989) Limb salvage in diabetics with foot ulcers. Prosthet Orthot Int 13:100-103

27. Viswanathan V, Madhavan S, Gnanasundaram S et al (2004) Effectiveness of different types of footwear insoles for the diabetic neuropathic foot: a follow-up study. Diabetes Care 27:474-477

28. Caravaggi C, De Giglio R, Pritelli C et al (2003) HYAFF 11-based autologous dermal and epidermal grafts in the treatment of noninfected diabetic plantar and dorsal foot ulcers: a prospective, multicenter, controlled, randomized clinical trial. Diabetes Care 26:2853-2859

29. Faglia E, Dalla Paola L, Clerici G et al (2005) Peripheral angioplasty as the first-choice revascularization procedure in diabetic patients with critical limb ischemia: prospective study of 993 consecutive patients hospitalized and followed between 1999 and 2003. Eur J Vasc Endovasc Surg 29:620-627

30. Adam DJ, Beard JD, Cleveland T et al (2005) Bypass versus angioplasty in severe ischaemia of the leg (BASIL): multicentre, randomised controlled trial. Lancet 366:1925-1934

31. Raymakers JT, Houben AJ, van der Heyden JJ, Tordoir JH, Kitslaar PJ, Schaper NC (2001) The effect of diabetes and severe 
ischaemia on the penetration of ceftazidime into tissues of the limb. Diabet Med 18:229-234

32. Harkless L, Boghossian J, Pollak R et al (2005) An open-label, randomized study comparing efficacy and safety of intravenous piperacillin/tazobactam and ampicillin/sulbactam for infected diabetic foot ulcers. Surg Infect (Larchmt) 6:27-40

33. Lipsky BA, Armstrong DG, Citron DM, Tice AD, Morgenstern DE, Abramson MA (2005) Ertapenem versus piperacillin/tazobactam for diabetic foot infections (SIDESTEP): prospective, randomised, controlled, double-blinded, multicentre trial. Lancet 366:1695-1703

34. Abbott CA, Carrington AL, Ashe H et al (2002) The North-West Diabetes Foot Care Study: incidence of, and risk factors for, new diabetic foot ulceration in a community-based patient cohort. Diabet Med 19:377-384

35. Litzelman DK, Marriott DJ, Vinicor F (1997) Independent physiological predictors of foot lesions in patients with NIDDM. Diabetes Care 20:1273-1278 